ARTICLE

Received 5 Mar 2013 | Accepted 29 Apr 2013 | Published 28 May $2013 \quad$ DOl: 10.1038/ncomms2942

\title{
Photo-oxidative enhancement of polymeric molecular sieve membranes
}

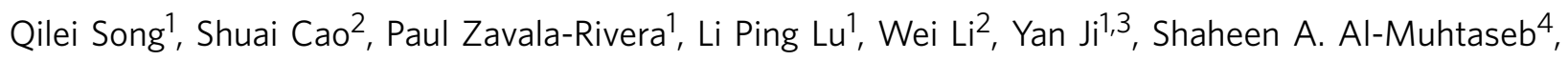
Anthony K. Cheetham ${ }^{2} \&$ Easan Sivaniah ${ }^{1}$

High-performance membranes are attractive for molecular-level separations in industrialscale chemical, energy and environmental processes. The next-generation membranes for these processes are based on molecular sieving materials to simultaneously achieve high throughput and selectivity. Membranes made from polymeric molecular sieves such as polymers of intrinsic microporosity (pore size $<2 \mathrm{~nm}$ ) are especially interesting in being solution processable and highly permeable but currently have modest selectivity. Here we report photo-oxidative surface modification of membranes made of a polymer of intrinsic microporosity. The ultraviolet light field, localized to a near-surface domain, induces reactive ozone that collapses the microporous polymer framework. The rapid, near-surface densification results in asymmetric membranes with a superior selectivity in gas separation while maintaining an apparent permeability that is two orders of magnitude greater than commercially available polymeric membranes. The oxidative chain scission induced by ultraviolet irradiation also indicates the potential application of the polymer in photolithography technology.

\footnotetext{
${ }^{1}$ Cavendish Laboratory, Department of Physics, University of Cambridge, Cambridge CB3 OHE, UK. ${ }^{2}$ Department of Materials Science and Metallurgy, University of Cambridge, Cambridge CB2 3QZ, UK. ${ }^{3}$ Department of Chemistry, Tsinghua University, Beijing 100084, China. ${ }^{4}$ Department of Chemical Engineering, Qatar University, PO Box 2713, Doha, Qatar. Correspondence and requests for materials should be addressed to E.S. (email: es10009@cam.ac.uk).
} 
$\mathrm{M}$ embrane-separation technology has become a promising alternative to conventional energy-intensive separation processes such as distillation or absorption, for example, in natural gas sweetening, hydrogen recovery and production, carbon dioxide separation from flue gas and air separation ${ }^{1}$. State-of-the-art polymeric gas-separation membranes are subject to a trade-off between permeability and selectivity, as theoretically predicted by Freeman ${ }^{2}$, also known as the Robeson's upper bound ${ }^{3-4}$. Research of the next-generation separation membrane is focused on tuning both local chemical interactions and pore sizes towards the kinetic diameter of molecules to achieve both selective separation and high throughput ${ }^{1,5-14}$. Over the past decade, significant progress has been made in molecular sieving materials such as carbon-based membranes (for example, carbon nanotubes ${ }^{15-16}$ and graphene ${ }^{9}$ ) and zeolites ${ }^{7}$. However, such membranes have not found commercial applications in gas separation because of high cost and scale-up impracticalities ${ }^{17}$. In contrast, microporous (pore sizes $<2 \mathrm{~nm}$ ) organic polymer materials are considered to have more impressive near-future impacts. Prospective classes of materials include metal-organic frameworks ${ }^{18}$, covalent organic frameworks ${ }^{19}$, substituted polyacetylenes, ${ }^{20}$ thermally rearranged polymer $^{6}$, conjugated microporous polymer ${ }^{21}$ and polymers of intrinsic microporosity (PIMs) $^{10-12,22-25}$. These microporous polymer materials have attracted significant interest for potential applications such as gas storage and separation, catalysis, energy storage and optoelectronics ${ }^{26}$.

Among these microporous polymer materials, solution-processable PIMs have emerged as promising candidates of membrane materials for applications in large-scale gas separation ${ }^{10-12,22-25}$. A typical example is PIM-1 (see Supplementary Fig. S1 for structure and synthesis) ${ }^{11}$. The polymer repeating unit is rigidly kinked via a spiro-carbon centre that orthogonally connects two planar aromatic units. The kink leads to inefficient packing of polymer chains and a high fractional free volume. PIM class materials behave as molecular sieves (PIM-1 has a nominal pore size of $<2 \mathrm{~nm}$ ) and have extremely high gas permeability (for example, for PIM-1, permeability of $\mathrm{CO}_{2} P_{\mathrm{CO} 2} \sim 5,000$ Barrer, 1 Barrer $=1 \times 10^{-10} \mathrm{~cm}^{3}(\mathrm{STP}) \mathrm{cm} \mathrm{cm}^{-2} \mathrm{~s}^{-1} \mathrm{cmHg}^{-1}$ ) but only a moderate selectivity, $\alpha$, which is defined as the relative permeability of one gas species to another. Hence, the selectivity of carbon dioxide over nitrogen and methane for PIM-1 are $\alpha_{\mathrm{CO} 2 / \mathrm{N} 2} \sim 15-20$ and $\alpha_{\mathrm{CO} 2 / \mathrm{CH} 4} \sim 10$, respectively, while the selectivity of oxygen over nitrogen is $\alpha_{\mathrm{O} 2 / \mathrm{N} 2} \sim 3-4$. In contrast, industrially used polymeric gas-separation membrane materials are much less permeable but achieve better selectivity (for example, for polyimide membranes, $P_{\mathrm{CO} 2}$ is $\sim 10$ Barrer, and $\alpha_{\mathrm{CO} 2 / \mathrm{CH} 4}$ is $\sim 30$ ).

Currently, there are several strategies to improve the separation performance of PIMs membranes: (i) enhancing the rigidity of polymer chain by synthesis of new monomers ${ }^{12,27-30}$, (ii) postsynthetic modification of PIMs polymer ${ }^{10,25,31}$, (iii) mixed matrix membranes ${ }^{32-34}$ and (iv) crosslinking induced by chemical ${ }^{35}$ or heat treatment ${ }^{36}$. Ultraviolet light irradiation is a widely used technique for processing of polymer materials ${ }^{8,37-40}$, for example, polymerization of novel gas-separation membranes ${ }^{40}$, creating nanostructures from block copolymer ${ }^{8}$, or surface modification of polymeric films (for example, polyimides and polydimethylsiloxane) ${ }^{8,37-39}$. Enhancement of membrane-separation properties by ultraviolet surface modification is not uncommon. In fact, it has been used since the early start of polymer membrane-separation industry in the 1980s (ref. 41), for example, in crosslinking polyimide membranes containing benzophenone units ${ }^{42-43}$ or degradation of siloxane-containing polymers ${ }^{44}$. However, the gas permeability of these conventional polymers is considerably low ${ }^{45}$. For the microporous PIMs polymers, understanding of the photooxidation phenomenon and stability is of broad interest to their wide range of applications (for example, gas separation, catalysis, optoelectronics and sensor).

In this study, using PIM-1 as a prototype of microporous polymers, we demonstrate that oxidative chain scission of PIM-1 polymer occurs upon short-wavelength ultraviolet irradiation in the presence of oxygen. Owing to the limited penetration of ultraviolet light and ozone, the oxidative chain scission is restricted to the surface and led to a novel near-surface collapse and densification, which is especially pronounced in such open microporous polymeric materials. The resulting asymmetric membranes exhibit a superior selectivity in gas separation while maintaining an apparent permeability that is two orders of magnitude greater than commercially available polymeric membranes. The rapid transformation of the near-surface physical and chemical properties via this photo-oxidative technique suggests further applications in other thin-film technologies.

\section{Results}

Photo-oxidation and characterization of polymer films. The ultraviolet irradiation $(\lambda=254 \mathrm{~nm})$ of PIM-1 membranes in the presence of oxygen resulted in the asymmetric changes of membrane morphology. Cross-sectional scanning electron microscopy (SEM) image (Fig. 1a) shows the post-ultraviolet asymmetric physical changes at the surface of a $50-\mu \mathrm{m}$-thick membrane where a dense skin layer was formed above a more mesoporous intermediate layer (pore size about $2-20 \mathrm{~nm}$, thickness about $0.1-2 \mu \mathrm{m}$ ). This intermediate layer was formed owing to the strain induced from densification of the skin layer; the remainder of the film was pristine PIM-1. The dense skin layer (Fig. 1b), forming quickly to a thickness of $300 \mathrm{~nm}$ within $10 \mathrm{~min}$ of ultraviolet exposure, thickened to $400 \mathrm{~nm}$ at longer exposure time (up to $60 \mathrm{~min}$ ) (Supplementary Figs S2 and S3). An identical trend was observed in 1.2- $\mu \mathrm{m}$-thin films (Fig. 1c). Figure 1c includes a superimposed plot of the transmitted intensity of ultraviolet light, $I(x)$, through the depth, $x$, of the film. Described by a Beer-Lambert expression as $I(x)=I_{0} \exp \left(-x / L_{0}\right)$, the transmitted intensity decreased significantly from $8 \mathrm{~mW} \mathrm{~cm}^{-2}$ to $40 \mu \mathrm{W} \mathrm{cm}{ }^{-2}$ at the depth of $500 \mathrm{~nm}$, reflecting a characteristic penetration depth $\left(L_{0}\right)$ of $\sim 105 \mathrm{~nm}$ for fresh PIM-1. This large attenuation in intensity comes from the fluorescent nature of PIM-1, which has an initial photoluminescence quantum yield efficiency of 37\% (Supplementary Fig. S4). The resulting surface-localized high ultraviolet field is responsible for much of the significant structural changes reported here.

Mechanical properties. During preparation of cross-sectional samples, very occasionally poorly fractured edges (Fig. 1d) indicated the contrast in mechanical properties of the skin and pristine regions. Nanoindentation measurements of the elastic modulus $(E)$ and the hardness $(H)$ were obtained on membranes at different conditions of ultraviolet irradiation using a sharp Berkovich tip in the continuous stiffness measurement mode (Supplementary Fig. S5 and Supplementary Table S1). The representative hardness profiles with depth of membranes $(0-500 \mathrm{~nm})$ are shown in Fig. 1e. The pristine PIM-1 film with thickness of $\sim 1 \mu \mathrm{m}$ gave the overall Young's modulus of $\sim 6.94 \mathrm{GPa}$ and hardness of $\sim 0.23 \mathrm{GPa}$. After ultraviolet irradiation for $60 \mathrm{~min}$ in air, we found $\sim 35 \%$ and $\sim 50 \%$ increases of both Young's modulus $(9.00-9.33 \mathrm{GPa})$ and hardness $(0.34-0.35 \mathrm{GPa})$ respectively. Equally it was found, from helium pycnometric studies, that the average density of the skin layer was $11 \%$ higher than the pristine PIM-1 film (Supplementary Methods).

Molecular weight distribution. Post-ultraviolet-treated membranes $(50 \mu \mathrm{m}$ thick) and thin films $(100-1,000 \mathrm{~nm})$ remained 

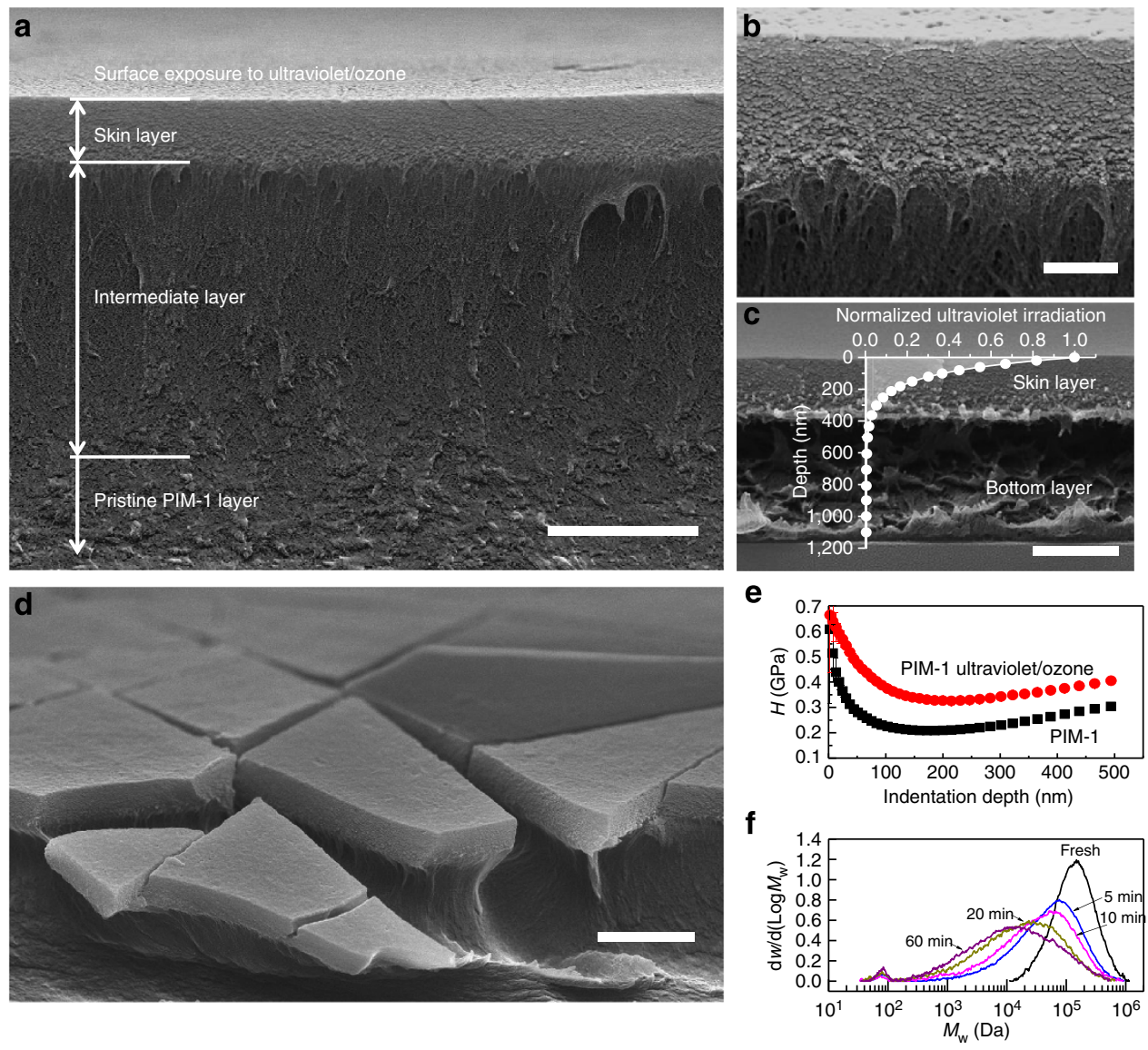

Figure 1 | Photo-oxidation of PIM-1 membranes. (a) Cross-sectional SEM of polymer of intrinsic microporosity (PIM-1) membrane ( 50 $\mu$ m thickness) after exposure to ultraviolet irradiation in air for $60 \mathrm{~min}$, showing the formation of composite layers consisting of a denser skin layer and an intermediate mesoporous layer over the pristine dense PIM-1 layer. (b) Enlargement of cross-sectional SEM of surface layer. (c) Cross-sectional SEM of ultraviolet/ ozone treated PIM-1 thin film. Inset shows the profile of ultraviolet light transmission as a function of depth. (d) Cross-sectional SEM of a fractured ultraviolet/ozone-treated PIM-1 membrane. Scale bar, $1 \mu \mathrm{m}$ (in a, d); $200 \mathrm{~nm}$ (in b); $500 \mathrm{~nm}$ (in c). (e) Harness $(H$ ) as a function of indentation depth of pristine and ultraviolet/ozone-treated PIM-1 film. (f) Evolution of molecular weight distribution of PIM-1 thin film ( $~ 300 \mathrm{~nm}$ ) exposure to ultraviolet irradiation in constant flow of pure $\mathrm{O}_{2}\left(100 \mathrm{ml} \mathrm{min}^{-1}\right)$ at different time.

soluble in solvents (for example, chloroform or tetrahydrofuran), confirming that ultraviolet treatment did not result in crosslinking of polymer. Instead, gel permeation chromatography (GPC) measurements with PIM-1 thin film $(\sim 300 \mathrm{~nm})$ showed that the average molecular weight decreased significantly. The pristine PIM-1 polymer has an average molecular weight of $M_{\mathrm{n}}=100 \mathrm{kDa}$ with a polydispersity index $\left(\mathrm{PDI}=M_{\mathrm{w}} / M_{\mathrm{n}}\right)$ of 1.8 . After ultraviolet irradiation in the presence of oxygen, the mean molecular weight decreases sharply indicating a broad range of fragmented polymer chains (Fig. 1f). However, the high PDI $(\sim 20)$ of the irradiated material limits the accuracy of characterizing the material with a single average molecular weight. This is to be expected from the attenuated ultraviolet light transmission with the depth of the thin film (Fig. 1c). The general observation of chain fragmentation was seen when samples were irradiated in air directly or through quartz glass (Supplementary Fig. S6). These degraded fractions also became soluble in polar solvents (for example, dimethylformamide (DMF), acetone or methanol). In principle, such degradation can limit the gasseparation applications of such ultraviolet/ozone-modified PIM-1 films in environments with extended exposure to condensable organic vapours. However, we expect that further post-treatment of this selective surface layer can stabilize it against chemical erosion.
Proposed mechanism of photo-oxidation. It is well known that under short-wavelength ultraviolet irradiation, $\mathrm{O}_{2}$ can absorb photons generating strongly oxidizing singlet oxygen and ozone. Equally, the high free volume and permeability of PIM-1 should lead to a local abundance of $\mathrm{O}_{2}$. Therefore, the PIMs materials behave as nanoreactors that are attacked by the oxidants generated within them, especially in the region of shallowly penetrating ultraviolet irradiation. We performed control experiments on ultraviolet irradiation of PIM-1 films in air, with films sandwiched between quartz glasses, which limited extent of oxygen exposure at the interface and carried out extensive characterization analyses. The conclusion of photo-oxidation and chain scission was essentially the same as those tests performed in air, or under gas flow with varied $\mathrm{O}_{2}$ concentration. Oxidative chain scission occurred as schematically shown in Fig. 2a. The oxidation is supported by Fourier Transform infrared spectroscopy analysis indicating formation of carbonyl and hydroxyl groups in ultraviolet/ozone-treated samples (Supplementary Fig. S7). Currently, the initial reaction site is still not clear and merits further study. The fragmented polymer segments pack more efficiently leading to loss of accessible free volume. This assumption was explored in molecular simulations of both PIM-1 (Fig. 2b) and the fragmented polymer (Fig. 2c). The accessible free volume, as probed by $\mathrm{CO}_{2}$ molecules with a kinetic diameter of $3.3 \AA$ at $298 \mathrm{~K}$, was 


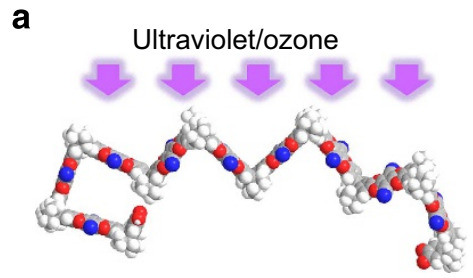

b

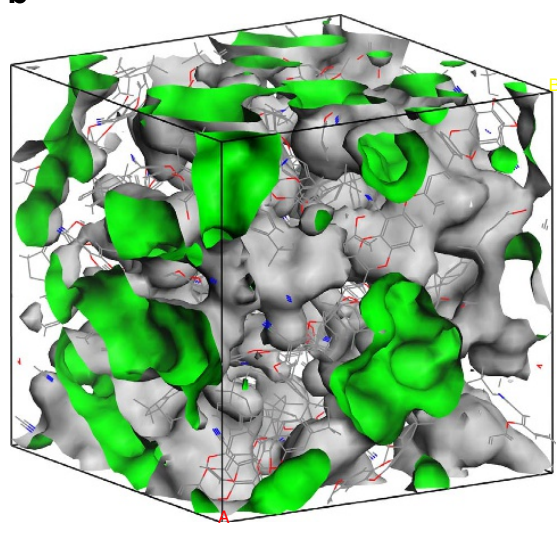

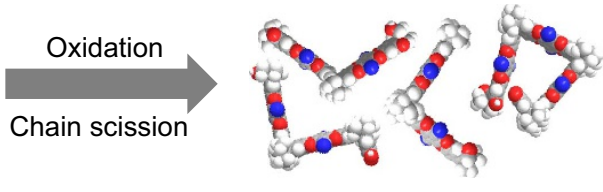

C

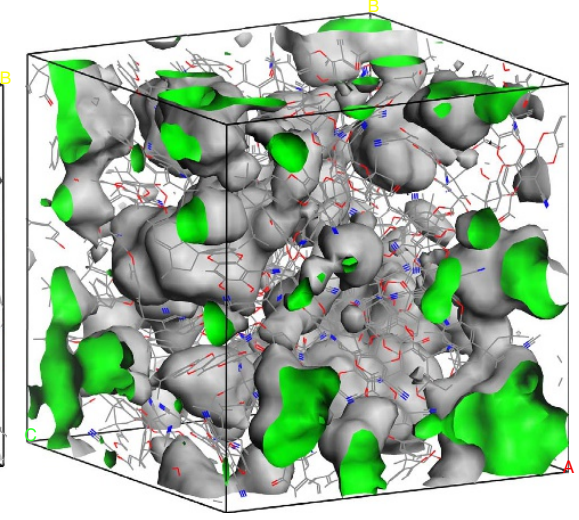

Figure 2 | Molecular structural transformation and simulation. (a) Schematic illustration of oxidative chain scission of PIM-1 upon exposure to ultraviolet irradiation in the presence of $\mathrm{O}_{2}$, showing transformation of long contorted polymer chains to shorter chains after oxidation and scission. (b) Threedimensional view of computer simulation of an amorphous cell with PIM-1 polymer chains (four polymer chains inside, each containing of ten repeating units). Cell size: $31.8 \times 31.8 \times 31.8 \AA$, density of $\sim 1.1 \mathrm{~g} \mathrm{~cm}^{-3}$. (c) Three-dimensional view of computer simulation of an amorphous cell with fragmented PIM-1, 20 polymer chains with two repeating PIM-1 units with oxidized groups, to mimic the shorter polymer chains after oxidative chain scission. Cell size: $29.5 \times 29.5 \times 29.5 \AA$, density of $\sim 1.3 \mathrm{~g} \mathrm{~cm}^{-3}$. The grey surface indicates the van der Walls surface and green surface is the Connolly surface with probe radius of $1.65 \AA$.

diminished in the simulation of densely packed shorter polymer chains.

Gas sorption properties. $\mathrm{N}_{2}$ adsorption at $77 \mathrm{~K}$ was performed with pristine PIM-1 thin films $(\sim 300 \mathrm{~nm})$ and modified films after exposure to ultraviolet irradiation in air for $20 \mathrm{~min}$. As shown in Fig. 3a, both pristine PIM-1 thin film and ultraviolet/ ozone-treated PIM-1 thin film exhibited high sorption at very low relative pressure, which is typical of microporous polymer. Both samples show broad distributions of pore size as derived from the non-local density functional theory (NLDFT) method (Supplementary Fig. S8). However, the ultraviolet/ozone-treated sample shows reduced pore volume over the range of $0.6-0.7 \mathrm{~nm}$. This loss of microporosity resulted in the decrease of BET surface area from 779 to $573 \mathrm{~m}^{2} \mathrm{~g}^{-1}$ and the micropore volume drops from 0.136 to $0.105 \mathrm{~cm}^{3} \mathrm{~g}^{-1}$. Similarly, we also observed a slight loss of $\mathrm{CO}_{2}$ solubility in the ultraviolet/ozone-treated PIM-1 films (Fig. 3a).

Gas transport properties. We varied the concentration of $\mathrm{O}_{2}$ in the ultraviolet irradiation atmosphere at a fixed exposure time (20 $\mathrm{min}$ ) and found significant differences in pure gas permeation properties of PIM-1 membrane and ultraviolet/ozone-modified PIM-1, as shown in Fig. 3b-c. Pristine PIM-1 membrane presents a $\mathrm{CO}_{2}$ permeability of $\sim 4,500$ Barrer, a $\mathrm{CO}_{2} / \mathrm{N}_{2}$ selectivity of $\sim 20$ and a $\mathrm{CO}_{2} / \mathrm{CH}_{4}$ selectivity at $\sim 13$, which are in agreement with the literature data ${ }^{46}$. Control experiments of ultraviolet exposure in pure $\mathrm{N}_{2}$ showed no discernible difference in properties to that of pristine polymer. In contrast, increasing the concentration of $\mathrm{O}_{2}$ (from 20.9 vol.\% in air to pure $\mathrm{O}_{2}$ ) enhanced the apparent selectivity of various gas pairs (for example, $\alpha_{\mathrm{CO} 2 / \mathrm{N} 2}$ and $\alpha_{\mathrm{CO} 2 / \mathrm{CH} 4}$ increased up to $\sim 30$ ) at the slight expense of permeability, corroborating the idea that ultravioletinduced ozone within the open polymer network is responsible for subsequent structural densification. As practical applications of such membranes in separations involve mixtures of gases, the mixed gas selectivity of important gas pairs (such as $\mathrm{CO}_{2} / \mathrm{CH}_{4}$ and $\mathrm{CO}_{2} / \mathrm{N}_{2}$ ) was determined from equimolar gas mixtures indicating a significant enhancement in separation efficiency in ultraviolet-treated membrane (as seen for the $\mathrm{CO}_{2} / \mathrm{CH}_{4}$ pair in Fig. 3d and also the $\mathrm{CO}_{2} / \mathrm{N}_{2}$ gas pair in Supplementary Fig. S9). Moreover, these tests performed with clean cylinder mixed gas indicated the physical stability of the modified membranes at high pressure.

We examined the dynamic change of gas transport properties of membranes exposed to ultraviolet irradiation in air for varied time of 5-60 min (for membranes exposed to air directly or membranes exposed to ultraviolet irradiation in air while sandwiched in quartz glass). Whether the membranes were exposed to air directly or sandwiched in quartz glass, we always observed the formation of densified skin layer (Supplementary Fig. S3) and the decrease in molecular weight of the exposed PIM-1 (Supplementary Fig. S6), confirming that the oxygen adsorbed within the polymer matrix was sufficient to induce the oxidation. However, sandwiching the membranes limited the source of $\mathrm{O}_{2}$ at the interface and consequently slightly affected the reaction kinetics and resulting gas transport properties (Supplementary Fig. S10 and Supplementary Table S2) without altering the general trend of lower permeability/higher selectivity for extended ultraviolet exposure. Here, we present the gas transport data of membranes exposed to ultraviolet irradiation in air, with the membranes sandwiched in quartz glass, as shown in Fig. 3e. Upon exposure to ultraviolet irradiation, the apparent permeability of $\mathrm{CO}_{2}$ showed a slight increase (up to 7,000 Barrer) after short exposure for 5-10 min, but then decreased with 
a

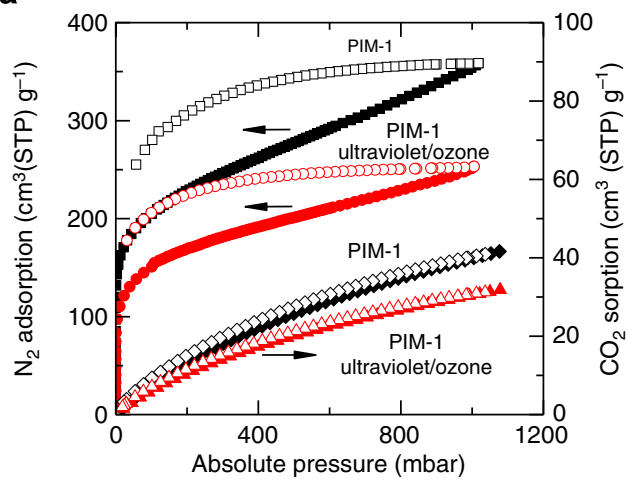

d

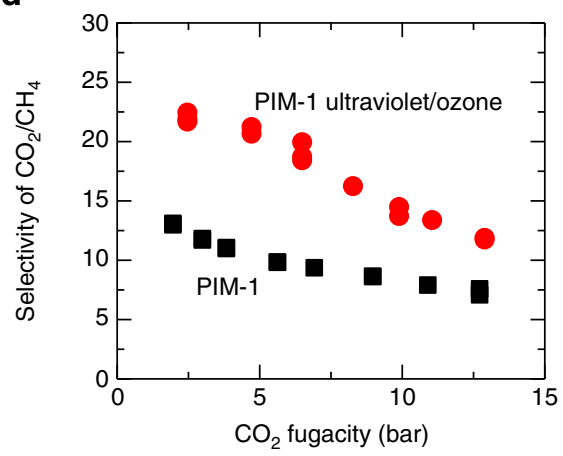

f

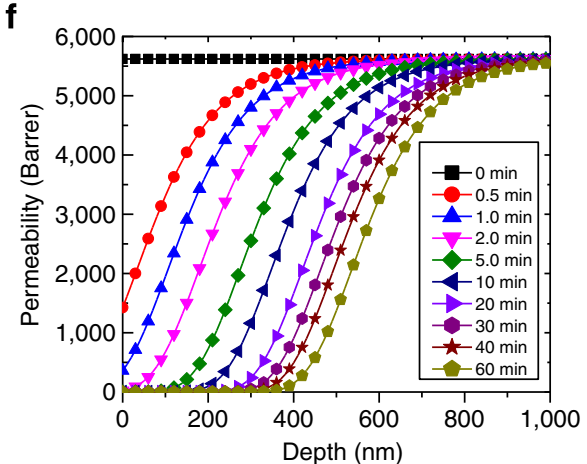

b

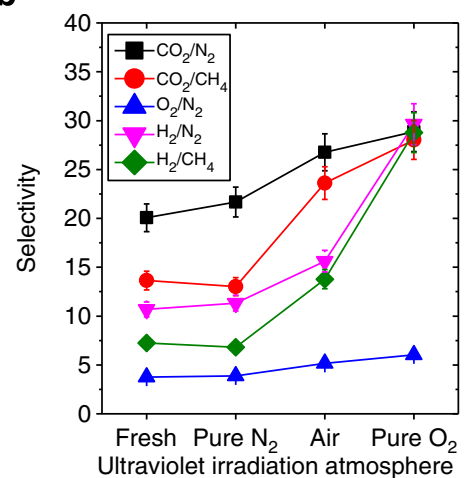

C

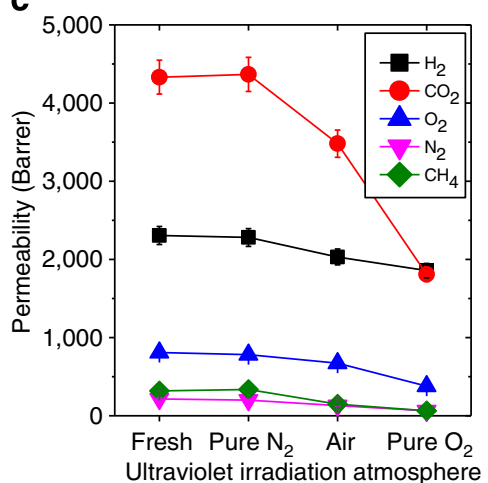

e

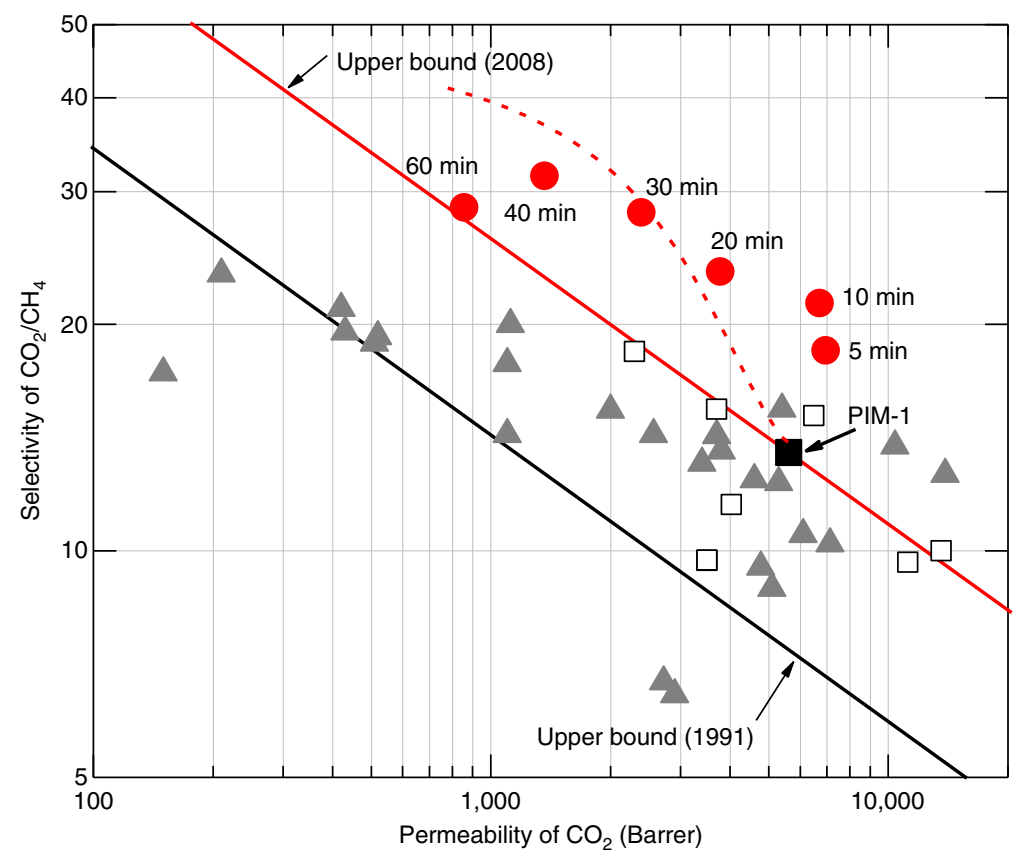

Figure 3 | Gas sorption and transport properties. (a) $\mathrm{N}_{2}$ adsorption isotherms at $77 \mathrm{~K}$ and $\mathrm{CO}_{2}$ sorption isotherms at $293 \mathrm{~K}$. Ultraviolet/ozone-modified PIM-1 was prepared by ultraviolet irradiation of PIM-1 thin film $(\sim 300 \mathrm{~nm})$ in air for $20 \mathrm{~min}$. Solid and open symbols represent the adsorption and desorption branch, respectively. (b) Apparent selectivity and (c) gas permeability of pristine and ultraviolet-irradiated (20 min, $20^{\circ} \mathrm{C}$ ) PIM-1 membranes ( $~ 50$ um) under different gas atmospheres. (d) Apparent selectivity of $\mathrm{CO}_{2} / \mathrm{CH}_{4}$ of PIM-1 membrane (solid squares) and ultraviolet/ozone-treated (30 min) PIM-1 membrane (solid circles) as a function of fugacity of $\mathrm{CO}_{2}$ in the mixed gas $\left(\mathrm{CO}_{2} / \mathrm{CH}_{4}, 50 / 50\right.$ vol.\%). (e) Apparent $\mathrm{CO}_{2} / \mathrm{CH}_{4}$ selectivity versus apparent $\mathrm{CO}_{2}$ permeability for PIM-1 (solid square) and ultraviolet/ozone-modified PIM-1 membranes (solid circles). The data are measured from pure-gas permeation tests with each gas repeated over three cycles of vacuum and permeation. The dashed line corresponds to model prediction. The dense membranes $(\sim 50 \mu \mathrm{m})$ were exposed to ultraviolet irradiation in air for varied time (5-60 min) as marked in the figure, with the membranes sandwiched in quartz glass. The open squares correspond to the literature data of PIM-1, the solid triangles represent the literature data of other PIMs, as summarized in a review by Du et al..$^{46}$ and data reported recently by Bezzu et al..$^{30}$ and Carta et al. ${ }^{12}$ The upper bounds were summarized by Robeson in $1991^{3}$ and $2008^{4}$, respectively. (f) Prediction of transient intrinsic permeability of $\mathrm{CO}_{2}$ along the depth of PIM-1 membrane upon exposure to ultraviolet irradiation.

extended exposure for 20-60 min, while the selectivity evidently increased. The slight increase of permeability after short exposure (5-10 min) of ultraviolet irradiation was within the error of measurement and subjected to plasticization and physical ageing during the history of measurement (multiple cycles of vacuum and permeation of several gases). It can effectively be considered a transient effect that becomes less significant when considered over the overall trends of permeability-selectivity performance in several sets of gas pair as shown later in Fig. 4. The apparent selectivity of $\mathrm{H}_{2}$ over each of $\mathrm{N}_{2}$ and $\mathrm{CH}_{4}$ increased up to $\sim 60$ with moderate decrease of permeability of $\mathrm{H}_{2}(\sim 2,000$ Barrer $)$.
The apparent permeability of $\mathrm{O}_{2}$ decreased to 600 Barrer with an $\mathrm{O}_{2} / \mathrm{N}_{2}$ selectivity of $\sim 6$ (Supplementary Table S2 and Supplementary Fig. S10). The overall membranes have apparent selectivity of commonly used gas-separation membrane materials while retaining orders-of-magnitude higher permeability.

The apparent gas transport properties of ultraviolet/ozonemodified membranes exceed the limitations defined by Robeson's 2008 upper bound (Fig. 3e). However, it should be noted that as the membranes became asymmetric after modification, these measured permeability and selectivity are overall properties of the heterogeneous membrane. The Robeson's upper-bound 

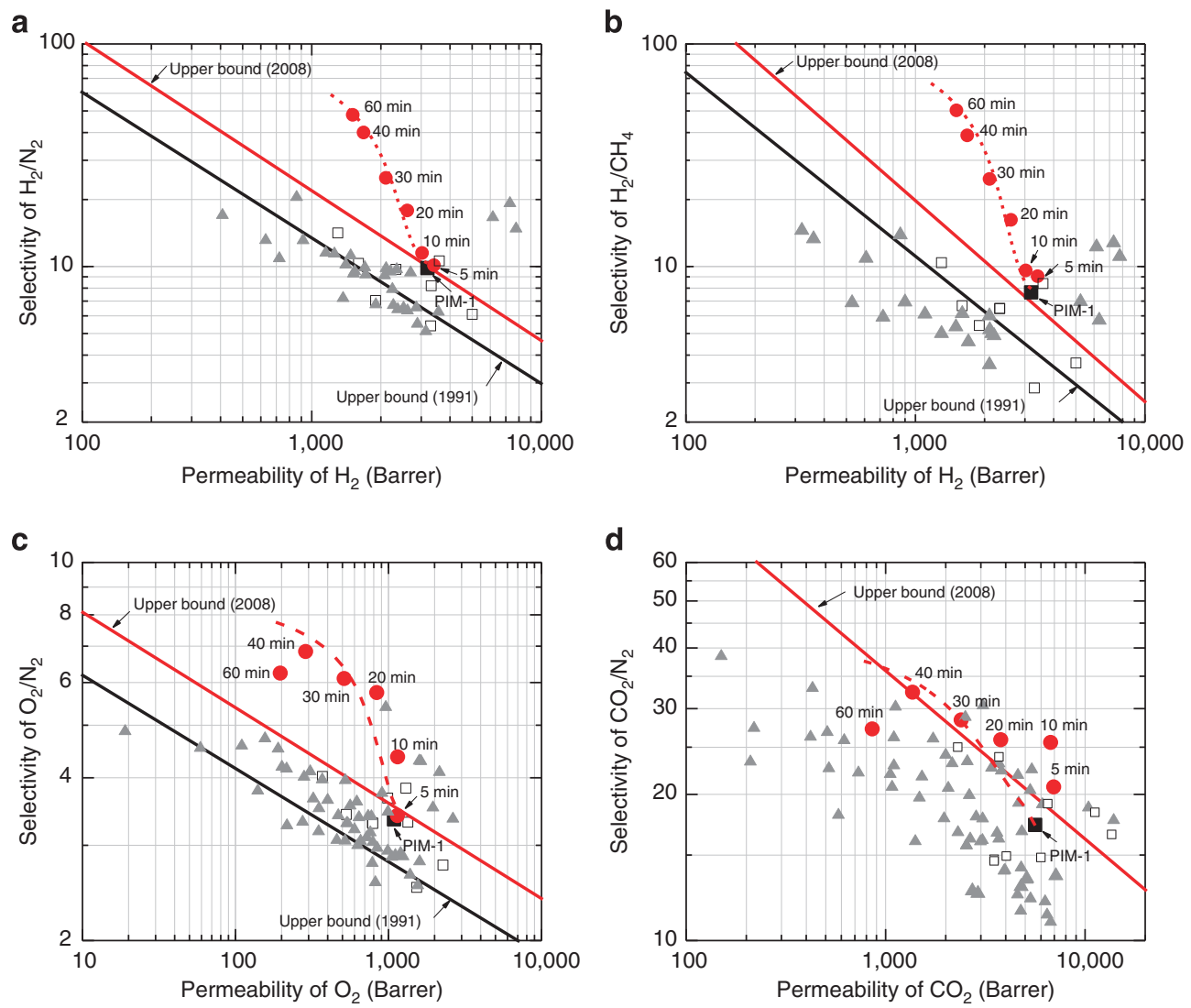

Figure 4 | Apparent gas transport properties. Apparent selectivity versus apparent permeability of PIM-1 and ultraviolet/ozone-modified PIM-1 membranes for industrially important gas pairs (a) $\mathrm{H}_{2} / \mathrm{N}_{2}$, (b) $\mathrm{H}_{2} / \mathrm{CH}_{4}$, (c) $\mathrm{O}_{2} / \mathrm{N}_{2}$ and (d) $\mathrm{CO}_{2} / \mathrm{N}_{2}$. Solid squares: PIM-1 membrane; solid circles: ultraviolet/ozone-modified PIM-1 membranes; dashed lines: model predictions (see Supplementary Note 1); solid lines: the upper bounds as summarized by Robeson in $1991^{3}$ and $2008^{4}$, respectively; the open squares correspond to the literature data of PIM-1, the solid triangles represent the literature data of other PIMs, as summarized in a review by Du et al. ${ }^{46}$ and data reported recently by Bezzu et al. ${ }^{30}$ and Carta et al. ${ }^{12}$ The dense membranes $(\sim 50 \mu \mathrm{m})$ were exposed to ultraviolet irradiation in air for varied time (5-60 $\mathrm{min}$ ) as marked in the figure, with the membranes sandwiched in quartz glass. The data are measured from pure-gas permeation tests at 4 bar at $22^{\circ} \mathrm{C}$.

construction, on the other hand, reflects the intrinsic permeability and selectivity of homogeneous dense polymer membranes. Thus, in a strict sense, one should be careful to make a comparison of the performance of our ultraviolet/ozone-modified membranes to the upper bound. However, in a field where it is becoming more common to generate composite materials for gas separation, whether through a bulk mixed matrix membranes, or as thin film composites (TFCs), the comparison is still instructive. This is effectively illustrated in modelling our observed results below, where we illustrate that the permeability and selectivity of the surface-modified PIM-1 can be predicted through a multi-layered model of varied intrinsic gas permeability so that the apparent 'bulk' values surpass the empirical upper-bound limitation of a homogeneous material, as shown in Figs $3 \mathrm{e}$ and 4.

The observed trends in selectivity and permeability as a function of ultraviolet irradiation time can be understood given that the exponentially decaying ultraviolet field generates a concentration gradient of ozone within the surface layer of the polymer. Making the broad assumption that ozone (and singlet oxygen) is responsible for the collapse of the PIM-1 framework, and hence the increase in local density and decrease in local permeability allows a determination of the overall and intrinsic permeability and selectivity of the membranes for different ultraviolet dosages. One such model calculation is given in Fig $3 \mathrm{e}$, making a further assumption of a first-order oxidative chain scission reaction (Supplementary Fig. S11 and Supplementary Note 1). An encouraging result of this model is the step-like depth-dependent permeability front that moves into the thin film over prolonged ultraviolet exposure time (as shown in Fig. 3f) over similar time and length scales to the observed skin-layer structures. The model also allows prediction of overall permeability and selectivity of other important gas pairs, as shown in Fig. 4. More details of this model will be provided in a future publication. However, it captures the essence of the observed structural changes but does not take into account the additional time-dependent effect of structural collapse on the diffusion of ozone in the polymer matrix. This explains why the model predicts a continued thickening of the skin layer into the bulk of the membrane (Fig. 3f) whereas in fact this slows down experimentally as the network above it collapses.

\section{Discussion}

In this study, the key contribution to the observed enhanced material properties arise from the role of ultraviolet-induced, internally generated oxidant in producing a dense selective surface skin to a high-permeability and porous material. The resulting asymmetric membrane geometry is analogous to TFC membranes. Nevertheless, from a chemical reaction viewpoint, the kinetics of photo-oxidation in PIM-1 could be tuned, that is, by controlling the intensity of ultraviolet irradiation, exposure time and oxygen concentration $\left[\mathrm{O}_{2}\right]$ within the polymer matrix. These parameters determine the concentration of intermediate species of ozone $\left[\mathrm{O}_{3}\right]$, singlet oxygen $[\mathrm{O}]$ and radicals within the 


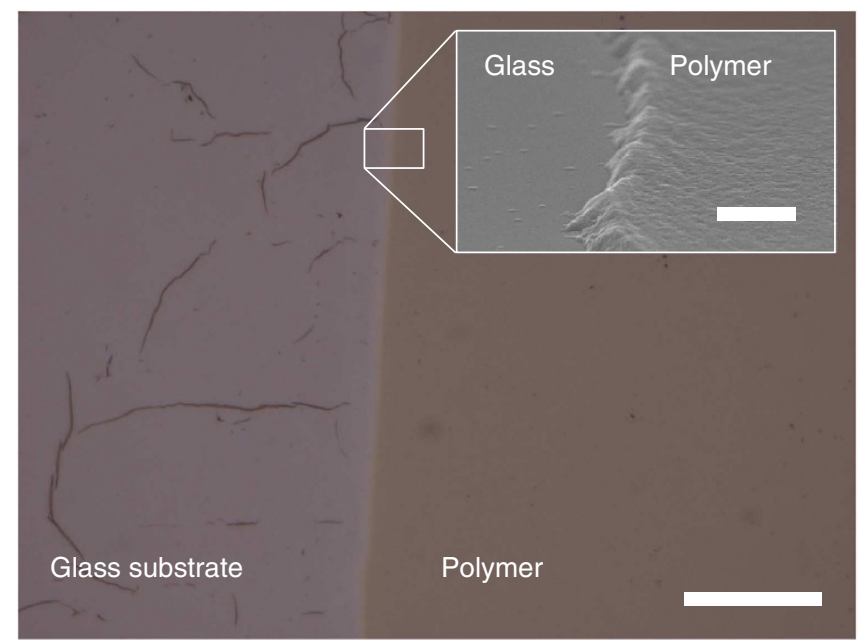

Figure 5 | Photoresist development. Optical microscopy image and SEM image (inset) of PIM-1 polymer thin film exposed to ultraviolet irradiation and wet etching with polar solvent. The PIM-1 polymer film (initial thickness of $300 \mathrm{~nm}$ ) was exposed to ultraviolet irradiation in air for $10 \mathrm{~min}$ with the right half of the film protected by an overlying cleaved silicon wafer used as a basic mask; followed with wet etching in a polar solvent, DMF, the ultraviolet-exposed area was dissolved away in DMF as indicated by the interface shown in the inset SEM image. Scale bar, $100 \mu \mathrm{m}$ (in optical image); $500 \mathrm{~nm}$ (in inset SEM).

polymer matrix. Therefore, it might be possible to scale-down the asymmetric pore structure on TFC membranes, to achieve higher selectivity while maintaining high permeance. As a rapid and simple processing technique, this implied modification of PIM-1 can be applied to various geometries of separation unit (for example, flat-sheet, spiral wound or hollow fibre configurations). Though the system has immediate application in dry gas atmosphere, the instability to condensable polar vapours solvents may initially limit its use in some industrial gas processing systems. For example, the membranes recovered the high permeability and low selectivity of fresh PIM-1 membranes after the ultraviolet/ozone-modified membrane had been immersed in polar solvents. This accelerated solvent test suggests that further post-treatment of the ultraviolet-modified surface is required to extend the applicability and endurance of the material. However, photo-oxidation introduces carbonyl and hydroxyl groups that can be used to stabilize the modified layer (for example, decarboxylation-induced crosslinking ${ }^{36}$, or thermal rearrangement ${ }^{6}$ ). Conversely, with a strong resonance to techniques used in photolithography, the ultraviolet-generated rapid structural phase change should also make the material amenable to wet- and dryetch photoresist and pattern development techniques. As a simple demonstration, PIM-1 films were exposed to ultraviolet light through a rudimentary edge-mask. A relatively clean, but unoptimized, topographical step profile was obtained after wet etching of the film with DMF (Fig. 5).

A previous patent by the UOP company found that ultraviolet irradiation of PIM-1 polymer resulted in higher selectivity and compromised permeability and attributed this performance, without any scientific proof, to ultraviolet crosslinking of PIM- $1^{47}$. During the writing of this paper, Chung and coworkers reported similar phenomenon of PIM-1 polymer membranes sandwiched between quartz plates and exposed to ultraviolet irradiation; they proposed a rearrangement mechanism at the spiro centre ${ }^{48}$. There are no significant differences in the measured gas permeability reported in such work and this paper. However, the attribution of the performance to crosslinking (in the UOP patent) or chain rearrangement by Chung and co-workers is clearly in error. Here, we proposed a photo-oxidative surface modification mechanism based on the evidence of our physical and chemical characterizations. Specifically, we observed the evident loss of PIM-1 molecular weight upon ultraviolet irradiation (in air or quartz-sandwiched samples), the limited ultraviolet transmission within the polymer and formation of surface-densified layers. These observations deny the possibility of a bulk material transformation. In ruling out these other explanations, and by revealing the true physical transformation at work, we can invoke a simple reactioncontrolled model of a surface-limited densification of the material. As seen by Figs $3 \mathrm{~d}$ and 4, the robustness of such a model is reinforced through its good predictions of apparent permeability in ultraviolet-transformed PIM-1 over a wide range of industrially important gas pairs.

In summary, photo-oxidation can be a simple and powerful method to enhance the gas-separation performance of high freevolume polymeric molecular sieve membranes. These materials, and indeed other microporous materials, have an intrinsically greater potential for structural collapse than conventional dense polymers. The tight surface attenuation, due in part to the strong photo-absorbance in PIM-1, allows the generation of a sharp near-surface structural phase transition within the first few hundred nanometres of the material in an eminently tuneable fashion. Such controlled surface modification should be available through other analogous chemistry and can be used beyond the discussed separation application, for example, in microfabrication and coating technologies.

\section{Methods}

Synthesis of polymer. The PIM-1 polymer was synthesized following the method described by Budd and McKeown ${ }^{22}$, and an optimized recipe by Guiver and co-workers ${ }^{49}$. A one-step polycondensation via a double aromatic nucleophilic

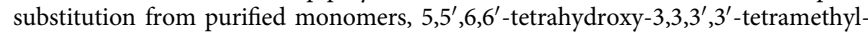
$1,1^{\prime}$-spirobisindane (TTSBI, $10.213 \mathrm{~g}, 30 \mathrm{mmol}$, Alfa Aesar) and 2,3,5,6tetrafluoroterephthalonitrile (TFTPN, $6.003 \mathrm{~g}, 30 \mathrm{mmol}$, Matrix Scientific), was performed in the presence of $\mathrm{K}_{2} \mathrm{CO}_{3}(8.292 \mathrm{~g}, 60 \mathrm{mmol}$, Aldrich) in anhydrous DMF ( $200 \mathrm{ml}$, Alfa Aesar). After the mixture has been stirred at $65^{\circ} \mathrm{C}$ for about $40 \mathrm{~h}$, the polymer was purified by dissolving in chloroform and reprecipitation from methanol, filtered and dried in vacuum oven at $110^{\circ} \mathrm{C}$ for overnight. The molecular weight was determined from GPC, giving an average molecular weight of $M_{\mathrm{n}}=100,000 \mathrm{Da}$ and a polydispersity (PDI) of 1.8 .

Preparation of membrane. Dense membranes were prepared by solution casting of filtered PIM-1 solution in chloroform on clean glass substrate, with solvent slow evaporated and removed. Thin films were prepared by spin coating or dip coating with diluted PIM-1 solution in chloroform.

Photo-oxidation. The membranes were exposed to ultraviolet irradiation $\left(\lambda=254 \mathrm{~nm}\right.$, incident intensity of $\left.8.0 \mathrm{~mW} \mathrm{~cm}^{-2}\right)$ in a commercial ultraviolet box (UVP model CX-2000). The membranes were exposed in air for exposure time studies. Alternatively, we controlled the atmosphere of ultraviolet irradiation using a temperature-controlled, quartz-windowed, stainless steel reactor with constant flow $\left(100 \mathrm{ml} \mathrm{min}^{-1}\right.$, STP) of different gases.

Characterization. SEM analysis of membranes (fractured in liquid nitrogen) was performed using a Hitachi S5500 microscope. The molecular weight was quantified by GPC calibrated with polystyrene standards. Fourier Transform infrared spectroscopy of PIM-1 thin films, dip coated on $\mathrm{NaCl}$ plates after exposure to ultraviolet irradiation, were measured with a NICOLET spectrometer (iS10, Thermo Scientific) in transmission mode. Density values of both as prepared PIM-1 membrane and ultraviolet/ozone-treated samples were measured using a Micromeritics Accupyc 1340 helium pycnometer. Thermal analyses were performed in a thermogravimetric analyser (TGA) Q600 (simultaneous TGA-DSC). Nitrogen adsorption-desorption measurements at $77 \mathrm{~K}$ and $\mathrm{CO}_{2}$ sorption at $293 \mathrm{~K}$ were carried out using a Micromeritics ASAP 2020 instrument. Nanoindentation was performed at ambient temperature using an MTS NanoIndenter ${ }^{\circledR}$ XP (MTS Corp., Eden Prairie, MN), equipped with the continuous stiffness measurement module. A three-sided pyramidal Berkovich tip was used to determine the Young's modulus $(E)$ and hardness $(H)$. 
Molecular simulation. For molecular modelling, energy minimization and molecular dynamics were performed with the Materials Studio software package (Version 4.3, Accelrys Inc., CA, USA). In one amorphous cell, four PIM-1 polymer chains with ten repeating units were constructed. To mimic the chain scission, 20 short chains containing two repeating units of PIM-1 with oxidized groups were constructed in another amorphous cell as well.

Optical spectroscopy methods. Ultraviolet-visible absorption spectra of PIM-1 thin film ( $\sim 100 \mathrm{~nm}$, as measured by SEM) were measured using a Hewlett Packard 8453 ultraviolet-Vis spectrometer, with an operating spectral range of $190-1,000 \mathrm{~nm}$. Steady-state photoluminescence excitation measurements were taken on CARY Eclipse fluorescence spectrophotometer, at an excitation wavelength of $420 \mathrm{~nm}$.

Gas permeation methods. Pure gas permeation tests were carried out at a feed pressure of $4 \mathrm{bar}$ and at temperature of $22^{\circ} \mathrm{C}$, using a constant volume pressure increase apparatus described elsewhere ${ }^{50}$. Mixed gas permeation was performed in another apparatus using the constant flow method, with certified gas mixtures of $\mathrm{CO}_{2} / \mathrm{N}_{2}\left(50 / 50\right.$ vol.\%) and $\mathrm{CO}_{2} / \mathrm{CH}_{4}(50 / 50 \mathrm{vol} . \%)$ at feed pressure up to $35 \mathrm{bar}$ at $22{ }^{\circ} \mathrm{C}$. The stagecut, defined by permeate flow rate to feed rate $\left(1,500 \mathrm{ml} \mathrm{min}^{-1}\right)$ was less than $1 \%$. The gas compositions were analysed by a gas chromatograph (GC-2014, Shimadzu).

\section{References}

1. Gin, D. L. \& Noble, R. D. Designing the next generation of chemical separation membranes. Science 332, 674-676 (2011).

2. Freeman, B. D. Basis of permeability/selectivity tradeoff relations in polymeric gas separation membranes. Macromolecules 32, 375-380 (1999).

3. Robeson, L. M. Correlation of separation factor versus permeability for polymeric membranes. J. Membr. Sci. 62, 165-185 (1991)

4. Robeson, L. M. The upper bound revisited. J. Membr. Sci. 320, 390-400 (2008).

5. Koros, W. J. \& Mahajan, R. Pushing the limits on possibilities for large scale gas separation: which strategies? J. Membr. Sci. 175, 181-196 (2000).

6. Park, H. B. et al. Polymers with cavities tuned for fast selective transport of small molecules and ions. Science 318, 254-258 (2007).

7. Lai, Z. et al. Microstructural optimization of a zeolite membrane for organic vapor separation. Science 300, 456-460 (2003).

8. Zavala-Rivera, P. et al. Collective osmotic shock in ordered materials. Nat. Mater. 11, 53-57 (2012).

9. Koenig, S. P., Wang, L., Pellegrino, J. \& Bunch, J. S. Selective molecular sieving through porous graphene. Nat. Nano. 7, 728-732 (2012).

10. $\mathrm{Du}, \mathrm{N}$. et al. Polymer nanosieve membranes for $\mathrm{CO}_{2}$-capture applications. Nat. Mater. 10, 372-375 (2011).

11. Budd, P. M. et al. Solution-processed, organophilic membrane derived from a polymer of intrinsic microporosity. Adv. Mater. 16, 456-459 (2004).

12. Carta, M. et al. An efficient polymer molecular sieve for membrane gas separations. Science 339, 303-307 (2013).

13. Cheetham, A. K., Férey, G. \& Loiseau, T. Open-framework inorganic materials. Angew. Chem., Int. Ed. 38, 3268-3292 (1999).

14. Merkel, T. C. et al. Ultrapermeable, reverse-selective nanocomposite membranes. Science 296, 519-522 (2002).

15. Hinds, B. J. et al. Aligned multiwalled carbon nanotube membranes. Science 303, 62-65 (2004).

16. Holt, J. K. et al. Fast mass transport through sub-2-nanometer carbon nanotubes. Science 312, 1034-1037 (2006).

17. Paul, D. R. Creating new types of carbon-based membranes. Science 335, 413-414 (2012).

18. Furukawa, H. et al. Ultrahigh porosity in metal-organic frameworks. Science 329, 424-428 (2010).

19. Côté, A. P. et al. Porous, crystalline, covalent organic frameworks. Science 310, 1166-1170 (2005).

20. Nagai, K. et al. Poly[1-(trimethylsilyl)-1-propyne] and related polymers: synthesis, properties and functions. Prog. Polym. Sci. 26, 721-798 (2001)

21. Cooper, A. I. Conjugated microporous polymers. Adv. Mater. 21, 1291-1295 (2009).

22. McKeown, N. B. \& Budd, P. M. Polymers of intrinsic microporosity (PIMs): organic materials for membrane separations, heterogeneous catalysis and hydrogen storage. Chem. Soc. Rev. 35, 675-683 (2006).

23. Budd, P. M. et al. Gas separation membranes from polymers of intrinsic microporosity. J. Membr. Sci. 251, 263-269 (2005).

24. McKeown, N. B. et al. Polymers of intrinsic microporosity (PIMs): Bridging the void between microporous and polymeric materials. Chemistry 11, 2610-2620 (2005)

25. Du, N. et al. High-performance carboxylated polymers of intrinsic microporosity (PIMs) with tunable gas transport properties. Macromolecules 42, 6038-6043 (2009).
26. Dawson, R., Cooper, A. I. \& Adams, D. J. Nanoporous organic polymer networks. Prog. Polym. Sci. 37, 530-563 (2012).

27. Du, N. et al. Polymers of intrinsic microporosity containing trifluoromethyl and phenylsulfone groups as materials for membrane gas separation. Macromolecules 41, 9656-9662 (2008).

28. Du, N., Robertson, G. P., Pinnau, I. \& Guiver, M. D. Polymers of intrinsic microporosity derived from novel disulfone-based monomers. Macromolecules 42, 6023-6030 (2009).

29. Du, N., Robertson, G. P., Pinnau, I. \& Guiver, M. D. Polymers of intrinsic microporosity with dinaphthyl and thianthrene segments. Macromolecules 43, 8580-8587 (2010).

30. Bezzu, C. G. et al. A spirobifluorene-based polymer of intrinsic microporosity with improved performance for gas separation. Adv. Mater. 24, 5930-5933 (2012).

31. Mason, C. R. et al. Polymer of intrinsic microporosity incorporating thioamide functionality: preparation and gas transport properties. Macromolecules 44, 6471-6479 (2011).

32. Ahn, J. et al. Gas transport behavior of mixed-matrix membranes composed of silica nanoparticles in a polymer of intrinsic microporosity (PIM-1). J. Membr. Sci. 346, 280-287 (2009).

33. Bushell, A. F. et al. Gas permeation parameters of mixed matrix membranes based on the polymer of intrinsic microporosity PIM-1 and the zeolitic imidazolate framework ZIF-8. J. Membr. Sci. 427, 48-62 (2013).

34. Bushell, A. F. et al. Nanoporous organic polymer/cage composite membranes. Angew. Chem., Int. Ed. 52, 1253-1256 (2012)

35. Du, N. et al. Azide-based cross-linking of polymers of intrinsic microporosity (PIMs) for condensable gas separation. Macromol. Rapid Commun. 32, 631-636 (2011).

36. Du, N., Dal-Cin, M. M., Robertson, G. P. \& Guiver, M. D. Decarboxylationinduced cross-linking of polymers of intrinsic microporosity (PIMs) for membrane gas separation. Macromolecules 45, 5134-5139 (2012).

37. Efimenko, K., Wallace, W. E. \& Genzer, J. Surface modification of sylgard-184 poly(dimethyl siloxane) networks by ultraviolet and ultraviolet/ozone treatment. J. Colloid Interface Sci. 254, 306-315 (2002).

38. Efimenko, K. et al. Nested self-similar wrinkling patterns in skins. Nat. Mater. 4, 293-297 (2005).

39. Maidenberg, D. A., Volksen, W., Miller, R. D. \& Dauskardt, R. H. Toughening of nanoporous glasses using porogen residuals. Nat. Mater. 3, 464-469 (2004).

40. Lin, H. et al. Plasticization-enhanced hydrogen purification using polymeric membranes. Science 311, 639-642 (2006).

41. Hayes, R. A. Polyimide gas separation membranes. US patent 4,717,393 (1988)

42. Kita, H., Inada, T., Tanaka, K. \& Okamoto, K. -i. Effect of photocrosslinking on permeability and permselectivity of gases through benzophenone- containing polyimide. J. Membr. Sci. 87, 139-147 (1994).

43. McCaig, M. S. \& Paul, D. R. Effect of UV crosslinking and physical aging on the gas permeability of thin glassy polyarylate films. Polymer (Guildf). 40, 7209-7225 (1999).

44. Park, H. B., Han, D. W. \& Lee, Y. M. Effect of a UV/ozone treatment on siloxane-containing copolyimides: surface modification and gas transport characteristics. Chem. Mater. 15, 2346-2353 (2003).

45. Yampolskii, Y. \& Freeman, B. D. Membrane Gas Separation (John Wiley \& Sons, Ltd, 2010).

46. Du, N., Park, H. B., Dal-Cin, M. M. \& Guiver, M. D. Advances in high permeability polymeric membrane materials for $\mathrm{CO}_{2}$ separations. Energy Environ. Sci. 5, 7306-7322 (2012).

47. Liu, C., Wilson, S. T. \& Lesch, D. A. UV-cross-linked membranes from polymers of intrinsic microporosity for liquid separations. US patent $7,758,751$ (2010).

48. Li, F. Y., Xiao, Y., Ong, Y. K. \& Chung, T.-S. UV-rearranged PIM-1 polymeric membranes for advanced hydrogen purification and production. Adv. Energy Mater. 2, 1456-1466 (2012).

49. Song, J. et al. Linear high molecular weight ladder polymers by optimized polycondensation of tetrahydroxytetramethylspirobisindane and 1,4dicyanotetrafluorobenzene. Macromolecules 41, 7411-7417 (2008).

50. Song, Q. L. et al. Zeolitic imidazolate framework (ZIF-8) based polymer nanocomposite membranes for gas separation. Energy Environ. Sci. 5, 8359-8369 (2012).

\section{Acknowledgements}

We are grateful to Professor William J. Koros and Professor Richard H. Friend for helpful suggestions. We acknowledge the financial support from an NPRP grant from the QNRF (Qatar) and the Engineering and Physical Sciences Research Council (EPSRC, UK). Q.S. acknowledges the China Scholarship Council. S.C. and A.K.C. acknowledge the European Research Council (ERC) and the Cambridge Overseas Trust. P.Z.-R. acknowledges the Consejo Nacional de Ciencia y Tecnología (CONACyT). We are grateful to the support from the Supercomputing Center of the Chinese Academy of Sciences. 


\section{Author contributions}

Q.S. and E.S. conceived the idea and designed the experiments. Q.S. performed major experiments, on synthesis of polymer, preparation of membrane, ultraviolet irradiation and optical measurements, gas permeation, molecular simulation, data analysis and interpretation, and wrote the paper; S.C., W. L. and A.K.C. performed gas sorption, density and TGA/DSC measurements, nanoindentation and data analysis; P.Z.-R. con tributed to the ultraviolet irradiation experiments; L.P.L. contributed to optical spectroscopy measurements and analysis; Y.J. contributed to synthesis of polymer; S.A.A. contributed to industrial application input; E.S. supervised the project and contributed to data analysis and interpretation, and to writing the paper. All authors discussed the results and commented on the manuscript.

\section{Additional information}

Supplementary Information accompanies this paper at http://www.nature.com/ naturecommunications

Competing financial interests: The authors declare no competing financial interests.

Reprints and permission information is available online at http://npg.nature.com/ reprintsandpermissions/

How to cite this article: Song, Q. et al. Photo-oxidative enhancement of polymeric molecular sieve membranes. Nat. Commun. 4:1918 doi: 10.1038/ncomms2942 (2013). 